\title{
Analytic continuation of Dirichlet series
}

\section{J. M. Anderson, D. Khavinson and H. S. Shapiro}

\section{Introduction}

The questions considered in this paper arose from the study [KS] of I. Fredholm's (insufficient) proof that the gap series $\sum_{0}^{\infty} a^{n} \zeta^{n^{2}}$ (where $0<|a|<1)$ is nowhere continuable across $\{|\zeta|=1\}$. The interest of Fredholm's method ([F], [ML]) is not so much its efficacy in proving gap theorems (indeed, much more general results can be got by other means, $c f$. the Fabry gap theorem in [Di]) as in the connection it made between certain special gap series and partial differential equations. For a full discussion of this see [KS]; here we shall only outline the salient points to provide motivation for a study of some function-theoretic questions that arise naturally when one tries to extend Fredholm's method to other kinds of gaps. As our starting point we take a slightly more general gap series than that of Fredholm, namely

$$
\varphi(\zeta)=\sum_{n=0}^{\infty} a_{n} \zeta^{n^{2}}
$$

where $\left\{a_{n}\right\}$ are complex and

$$
0<\varlimsup_{n \rightarrow \infty}\left|a_{n}\right|^{1 / n}<1 .
$$

Note that the radius of convergence is 1 (this would be so also under the weaker, and more natural condition where the right hand inequality 
in (1.2) is replaced by

$$
\varlimsup_{n \rightarrow \infty}\left|a_{n}\right|^{1 / n}<+\infty
$$

but the method to be employed is simpler when (1.2) is assumed). Now,

$$
u(z, w)=\sum_{n=0}^{\infty} a_{n} e^{n z+n^{2} w}
$$

is convergent to a holomorphic function for $(z, w) \in \mathbb{C} \times \mathcal{L}$, where

$$
\mathcal{L}=\{w \in \mathbb{C}: \operatorname{Re} w<0\}
$$

and satisfies

$$
\frac{\partial u}{\partial w}=\frac{\partial^{2} u}{\partial z^{2}}
$$

For real $z, w$ this is of course the "heat equation", with $w$ as the time variable, but here we consider the variables as complex. The initial value problem for (1.5) with data on $\left\{w=w_{0}\right\}$ is characteristic so, as S. Kovalevskaya already explained in her Habilitationsschrift [Ko], even holomorphic data $z \mapsto u\left(z, w_{0}\right)$ does not in general suffice to guarantee a local holomorphic solution of (1.5). (Weierstrass expressed great surprise at this result, and admiration for his pupil's discovery; $c f$. especially his letter to P. du Bois-Reymond of 15 December 1874, reproduced in Acta Math. 39). In fact, implicit in her reasoning is the following stronger statement: a solution to (1.5) holomorphic in a bidisk $D_{z} \times D_{w}$, where

$$
D_{z}=\left\{z:\left|z-z_{0}\right|<R_{1}\right\}, \quad D_{w}=\left\{w:\left|w-w_{0}\right|<R_{2}\right\}
$$

extends holomorphically to $\mathbb{C} \times D_{w}$. (This can nowadays be deduced from general theorems, $c f$. [Ki] or [BS], also [H, Theorem 9.4.8]. See also [KS] for a simple proof).

Fredholm misunderstood Kovalevskaya's result, interpreting it to imply that if, for a solution $u$ to (1.5) in the bidisk (1.6), the function $w \mapsto u\left(z_{0}, w\right)$ extends holomorphically across a boundary point $w_{1}$ of $D_{w}$, then $z \mapsto u\left(z, w_{1}\right)$ extends holomorphically to all of $\mathbb{C}$. This was the tool for Fredholm's attempt to prove the non-continuability of (1.1), and is (as shown in $[\mathrm{KS}]$ ) incorrect. We emphasize that the error lies 
in attempting to draw conclusions from the behaviour of $w \mapsto u\left(z_{0}, w\right)$ for just one value of $z_{0}$.

To "save" Fredholm's idea one can first establish the following refinement of the above-mentioned result of Kovalevskaya. We precede it with a convenient definition.

Definition. Let $f$ be a function of one complex variable, holomorphic on a neighborhood of $z_{0}$. Then, for $k \in \mathbb{N}$ the $k$-fold symmetrization of $f$ about $z_{0}$ is the function $t \mapsto F\left(t ; z_{0}, k\right)$ where

$$
F\left(t ; z_{0}, k\right)=\frac{1}{k} \sum_{j=0}^{k-1} f\left(z_{0}+\omega^{j} t\right), \quad \omega=e^{2 \pi i / k} .
$$

Note that $F$ is holomorphic on a neighborhood of $t=0$. The following is proven in [KS].

Theorem A. If $u$ is holomorphic on the bidisk (1.6) and satisfies (1.5) there, and $w \mapsto u\left(z_{0}, w\right)$ extends holomorphically to a neighborhood of a boundary point $w_{1}$ of $D_{w}$, then the 2-fold symmetrization of $z \mapsto$ $u\left(z, w_{1}\right)$ about $z_{0}$ extends to $\mathbb{C}$ as an entire function of order at most 2 .

For later purposes note that if (1.5) is replaced by

$$
\frac{\partial u}{\partial w}=\frac{\partial^{k} u}{\partial z^{k}}, \quad k \geq 3
$$

the corresponding conclusion holds for the $k$-fold symmetrization of $f$ about $z_{0}$.

Now we can apply Fredholm's idea correctly to show that $\varphi$ in (1.1) is not continuable across any point $\zeta=e^{i v_{0}}, v_{0} \in \mathbb{R}$. Indeed, if it were then, with $u$ given by $(1.4), u(0, w)$ would extend from $\mathcal{L}$ to a neighborhood of its boundary point $w_{0}=e^{i v_{0}}$ and so, by Theorem $\mathrm{A}$, the 2 -fold symmetrization about 0 of $\sum_{n=0}^{\infty} a_{n} e^{i n^{2} v_{0}} e^{n z}$ would extend as an entire function, that is

$$
\sum_{n=0}^{\infty} a_{n} e^{i n^{2} v_{0}}\left(e^{n z}+e^{-n z}\right)
$$

would extend from a neighborhood of $z=0$ to the entire $z$-plane without singularities. But, because of assumption (1.2) this is a Laurent 
series in $e^{z}$ with finite positive convergence radii. Since a Laurent series must have at least one singularity on each boundary circle of its annulus of convergence, we have a contradiction, and the noncontinuability of $\varphi$ in (1.1) is proved.

Mittag-Leffler's exposition [ML] of Fredholm's idea ends with the suggestion that the method employed can be applied to more general situations. Let us see what happens when we try to apply the (corrected) Fredholm method to showing that $\sum_{n=0}^{\infty} a_{n} \zeta^{n^{3}}$ is not continuable across any point of $\partial \mathbb{D}$, where again we assume (1.2) (since the gaps are bigger one might expect the proof to be easier, but the strangeness of the method is that it does not work this way, as we will see). Introduce again the variable change $\zeta=e^{w}$ and look at

$$
u(z, w)=\sum_{n=0}^{\infty} a_{n} e^{n z} e^{n^{3} w}
$$

which is holomorphic on $\mathbb{C} \times \mathcal{L}$ and satisfies

$$
\frac{\partial u}{\partial w}=\frac{\partial^{3} u}{\partial z^{3}}
$$

By the generalized form of Theorem $\mathrm{A}$, if $u(0, w)$ were continuable across a point $w=i v_{0},\left(v_{0} \in \mathbb{R}\right)$ of $\partial \mathcal{L}$, then the 3 -fold symmetrization of $z \mapsto u\left(z, i v_{0}\right)$ about 0 would be entire, i.e.

$$
\sum_{n=0}^{\infty} a_{n} e^{i n^{3} v_{0}}\left(e^{n z}+e^{\omega n z}+e^{\omega^{2} n z}\right),
$$

where $\omega=e^{2 \pi i / 3}$, would be entire. But, could this happen? Now (1.9) is no longer a Laurent series in $e^{z}$, but a Dirichlet series of quite general type: $\sum c_{m} e^{\lambda_{m} z}$ with complex exponents $\left\{\lambda_{m}\right\}$ lying on three rays through 0 . Even if (1.2) prevents the series from converging on the whole $z$-plane, there are no general theorems that rule out the analytic continuability of (1.9) to the whole plane. (Indeed, see [L] for discussion of phenomena which may occur).

It is fairly easy to show (see below, Section 4.2) that if we strengthen $(1.2)$ to

$$
0<\varlimsup_{n \rightarrow \infty}\left|a_{n}\right|^{1 / n} \leq c
$$

for a sufficiently small $c>0$, then (1.9) cannot extend to all of $\mathbb{C}$, and thus, in this case, we do obtain the noncontinuability of $\sum_{0}^{\infty} a_{n} \zeta^{n^{3}}$. 
But, perhaps surprisingly, the argument really fails essentially if only (1.2) is assumed. That is (and this is one of the main results of the present paper):

There exists complex $\left\{c_{n}\right\}_{n=0}^{\infty}$ with

$$
0<\varlimsup\left|c_{n}\right|^{1 / n}=\delta<1
$$

such that

$$
\sum_{n=0}^{\infty} c_{n}\left(e^{n z}+e^{\omega n z}+e^{\omega^{2} n z}\right)
$$

where $\omega=e^{2 \pi i / 3}$ (note that we have absolute convergence on a neighborhood of $z=0$ ) extends without singularities to all of $\mathbb{C}$. Indeed, the sum of this series can vanish identically.

An equivalent form of the last statement is obtained by evaluating the Taylor coefficients of (1.11) at $z=0$ :

There exist $\left\{c_{n}\right\}$ satisfying (1.10) such that

$$
\sum_{n=0}^{\infty} c_{n} n^{3 k}=0, \quad k=0,1,2, \ldots
$$

(where $0^{0}$ is interpreted as 1 ).

This formulation naturally leads to the consideration of the equations

$$
\sum_{n=0}^{\infty} c_{n} n^{p k}=0, \quad k=0,1,2, \ldots
$$

We shall show that solutions satisfying (1.10) exist for each $p>2$, but never for $p \leq 2$. Moreover, for $p>2$ there is no solution if $\delta<\delta_{p}$ where $\delta_{p}$ is sufficiently small, and for $p$ integral we shall find the best possible value of $\delta_{p}$. In the course of this work, certain other questions which arise naturally will also be discussed.

The rest of the paper is organized as follows. Section 2 deals with cases where (1.12) (and some more general equation systems) admits only the solution $c_{n}=0$. This is closely interwoven with known results concerning quasi-analytic functions. Section 3 contains our main 
result (Theorem 3.1) which shows the sharpness, in an important case, of the uniqueness theorem of Section 2; this example sheds light on the possibility of extending Fredholm's method to other kinds of gaps. In Section 4 it is shown that under certain conditions a function defined by a Dirichlet series of fairly general type cannot be analytically continued much beyond its domain of absolute convergence; this enables one to prove non-continuability of certain gap series by (a modification of) Fredholm's method. Section 5 contains a brief discussion of integral analogues of the problem treated in Sections 2 and 3; here fairly complete results are much easier to obtain.

\section{A uniqueness problem for Dirichlet series.}

Let us first consider a rather general situation, a Dirichlet series

$$
\sum_{n=1}^{\infty} c_{n} e^{\lambda_{n} z}
$$

where $\left\{\lambda_{n}\right\}$ and $\left\{c_{n}\right\}$ are complex. We may of course assume the $\lambda_{n}$ are pairwise distinct. From this point on various combinations of hypotheses could be made, some leading to uniqueness theorems and others not.

J. Wolff [W] constructed in 1921 examples that imply one can find $\left\{\lambda_{n}\right\}$ bounded and $\left\{c_{n}\right\}$ not all zero satisfying

$$
\sum_{n=1}^{\infty}\left|c_{n}\right|<+\infty
$$

and such that (2.1) (which then converges for all complex $z$ ) sums to 0 (however, Dirichlet series are not discussed in [W]). This is equivalent to finding a nontrivial solution $\left\{c_{n}\right\}$ satisfying (2.2) to the infinite system of linear equations

$$
\sum_{n=1}^{\infty} c_{n} \lambda_{n}^{k}=0, \quad k=0,1,2, \ldots
$$

Wolff's result is not given in terms of (2.3) but rather as the solution of a then long-standing uniqueness question concerning series of the type

$$
\sum_{n=1}^{\infty} \frac{c_{n}}{z-z_{n}}
$$


where $\left\{z_{n}\right\} \subset \mathbb{C}$. If $(2.2)$ holds, (2.4) converges uniformly on compact subsets of $\mathbb{C} \backslash K$, where $K$ denotes the closure of $\left\{z_{n}\right\}$, and various investigators (Borel, Carleman, Denjoy, Wolff, Beurling, ... ) have studied conditions under which (a) the "apparent singularities" $\left\{z_{n}\right\}$ of the sum (2.4) really are singular points for the sum function (which is analytic on each component of $\mathbb{C} \backslash K$ ), and (b) in case there is more than one component, the sum functions corresponding to different components are analytic continuations of one another. (e.g. Borel showed that (a) and (b) may fail if only (2.2) is imposed while they hold if $\varlimsup\left|c_{n}\right|^{1 / n}=0$.) The uniqueness problem for (2.4) is of course subsumed under (a). Henceforth we will not mention interpretations of our results involving series (2.4), but refer the reader to [BSZ] for this connection.

A. Beurling showed [Be, pp. 209-210] that a series (2.1) can converge everywhere to zero with bounded $\left\{\lambda_{n}\right\}$ and non-zero $\left\{c_{n}\right\}$ that satisfy not merely (2.2) but much stronger conditions, e.g.

$$
\left|c_{n}\right| \leq \exp \left(-n /(\log n)^{2}\right),
$$

whereas this is not possible if

$$
\varlimsup\left|c_{n}\right|^{1 / n}<1 .
$$

Returning to Dirichlet series (2.1), we will in the remainder of this section be considering cases where $\lambda_{n}>0$ and $\lambda_{n} \rightarrow \infty$. We begin with a basic uniqueness theorem. This is in principle known, as well as the corollaries we present; these results are scattered in the literature on quasi-analytic functions and Banach algebras. We need them to put in proper perspective the results of Section 3, and we include proofs for the reader's convenience.

Theorem 2.1. Let $0<\lambda_{1}<\lambda_{2}<\ldots$, and

$$
\varlimsup_{n \rightarrow \infty} \frac{(\log n)^{2}}{\lambda_{n}}=0 .
$$

Suppose, for some $\varepsilon>0$,

$$
\left|c_{n}\right| \leq e^{-\varepsilon \sqrt{\lambda_{n}}}
$$

If

$$
\sum_{n=1}^{\infty} c_{n} \lambda_{n}^{k}=0, \quad k=0,1,2, \ldots
$$

then all $c_{n}$ vanish. 
REMARK. This can be interpreted as a uniqueness theorem for series (2.1). Indeed, (2.6) and (2.7) imply that (2.1) as well as all its formally differentiated series converge absolutely for $\{z: \operatorname{Re} z \leq 0\}$, to some function $f$. Then $(2.8)$ is the assertion that $f$ and all its derivatives vanish at the boundary point 0 of this half-plane.

Before giving the proof, let us note some corollaries.

Corollary 1. ([Ca 2]). If $p>0$, and for some $\varepsilon>0$

$$
\left|c_{n}\right| \leq \exp \left(-\varepsilon n^{p / 2}\right)
$$

then

$$
\sum_{n=1}^{\infty} c_{n} n^{p k}=0, \quad k=0,1,2, \ldots
$$

implies $c_{n}=0$ for all $n$.

This is just the case $\lambda_{n}=n^{p}$ of the theorem, and much of the rest of this paper is devoted to the question of sharpness of the condition (2.9). A few cases follow from well known results.

First of all, look at the case $p=2$. The corollary says that if $\left\{c_{n}\right\}$ decay exponentially, and $\sum_{1}^{\infty} c_{n} n^{2 k}$ all vanish then all $c_{n}$ vanish. Here we certainly cannot weaken the hypothesis of exponential decay to, say

$$
\left|c_{n}\right| \leq \exp \left(-a n^{\alpha}\right)
$$

for some $\alpha<1$ since, as is well known from the theory of quasianalytic classes ( $c f .[\mathrm{M}]$ ), given $\alpha<1$, there is a nontrivial function $\sum_{n=1}^{\infty} c_{n} \cos n \theta$, where $\left\{c_{n}\right\}$ satisfies (2.11), for which all derivatives vanish at $\theta=0$, which is to say $\sum_{1}^{\infty} c_{n} n^{2 k}=0$ for $k=0,1,2, \ldots$ See also [Ha, p. $27 \mathrm{ff}$.] for a pioneering discussion in this vein.

Next, examine the case $p=1$. The corollary says that

$$
\left|c_{n}\right| \leq \exp \left(-\varepsilon n^{1 / 2}\right)
$$

and

$$
\sum_{1}^{\infty} c_{n} n^{k}=0, \quad k=0,1, \ldots
$$


imply that all $c_{n}$ vanish, or what is the same, (2.12) and the presence of an infinite order zero of $\sum_{1}^{\infty} c_{n} e^{i n \theta}$ at some $\theta_{0}$ imply all $c_{n}$ vanish. This is due to Carleson [Ca2]. Here again, one cannot weaken hypothesis (2.12), say to

$$
\left|c_{n}\right| \leq \exp \left(-b n^{\beta}\right)
$$

with $\beta<1 / 2$. Indeed, it can be shown that if $\beta<1 / 2$ the unique outer function $F_{\sigma}$ in the unit disk satisfying

$$
\left|F_{\sigma}\left(e^{i \theta}\right)\right|=\exp \left(-\left|\sin \frac{\theta}{2}\right|^{-\sigma}\right), \quad|\theta| \leq \pi
$$

where $\sigma<1$, has Taylor coefficients $\left\{c_{n}\right\}$ satisfying (2.14) if $\sigma=\sigma(\beta)$ is sufficiently close to 1 . (Again, $c f$. [Ha, pp. $27 \mathrm{ff}$.] for closely related material.)

In the next section we shall discuss the sharpness of (2.9) in some other, more delicate cases. We may remark (as we will see in Section 5) that for the integral analogue of these problems matters are much simpler: different values of $p$ are reducible to one another by a simple scaling argument (change of variables) but that is not possible with series. From a technical point of view, we stress that examples to show the sharpness of (2.9) are the main concern of this paper.

Corollary 2. ([Ca2]). If $f(z)=\sum_{n=1}^{\infty} c_{n} z^{n}$, where $\left\{c_{n}\right\}$ satisfy $(2.12)$, and $f$ has infinitely many zeroes in the open unit disk $\mathbb{D}$, then $f \equiv 0$.

Proof. By Corollary 1 it is enough to show $f\left(e^{i \theta}\right)$ has an infinite order zero at $\theta=\theta_{0}$, if $f$ vanishes at a sequence $\left\{z_{j}\right\} \subset \mathbb{D}$ with $\lim z_{j}=e^{i \theta_{0}}$. This is a well-known fact; we include the simple proof. It is based on

Lemma. [TW, Prop. 4.5]. If $f$ is analytic in $\mathbb{D}$ and its Taylor coefficients $\left\{a_{n}\right\}$ satisfy

$$
\left|a_{n}\right|=O\left(n^{-k}\right), \quad n \rightarrow \infty
$$

for every positive $k$ (or, what is the same, $f \in C^{\infty}(\overline{\mathbb{D}})$ ), and $f(\xi)=0$ for some $\xi \in \partial \mathbb{D}$, then $f(z)=(z-\xi) g(z)$ for some $g$ analytic in $\mathbb{D}$ and in $C^{\infty}(\overline{\mathbb{D}})$. 
Proof of Lemma. We may assume $\xi=1$. Write $f=\sum_{0}^{\infty} a_{n} z^{n}$, $g=\sum_{0}^{\infty} b_{n} z^{n}$ where $g=(1-z)^{-1} f$ is analytic in $\mathbb{D}$. Then,

$$
b_{n}=a_{0}+a_{1}+\cdots+a_{n}=-\left(a_{n+1}+a_{n+2}+\cdots\right)
$$

since $\sum_{0}^{\infty} a_{n}=f(1)=0$. Hence

$$
\left|b_{n}\right| \leq\left|a_{n+1}\right|+\left|a_{n+2}\right|+\cdots
$$

so that, using (2.15), also $\left\{b_{n}\right\}$ satisfies the estimates (2.15), hence $g \in C^{\infty}(\overline{\mathbb{D}})$ and the lemma is proved.

Deduction of Corollary 2. If $f$ vanishes at infinitely many points $\left\{z_{j}\right\}$ of $\mathbb{D}$ and $\xi \in \partial \mathbb{D}$ is a limit point of $\left\{z_{j}\right\}$ then $f(\xi)=0$, so $f=$ $(z-\xi) g(z)$ where $g \in C^{\infty}(\overline{\mathbb{D}})$. Now, $g\left(z_{j}\right)=0$, so $g(\xi)=0$ and hence $g=(z-\xi) h$ for some $h \in C^{\infty}(\overline{\mathbb{D}})$. Thus,

$$
f(z)=(z-\xi)^{2} h(z), \quad h \in C^{\infty}(\overline{\mathbb{D}}) .
$$

Continuing in this fashion we see that for each $m$ we have

$$
f(z)=(z-\xi)^{m} f_{m}(z)
$$

for a suitable $f_{m} \in C^{\infty}(\overline{\mathbb{D}})$. Thus, $f$ has a zero of infinite order at $\xi$, which completes the proof of Corollary 2.

REMARK. It is not hard to show that there are non-trivial functions analytic in $\mathbb{D}$ whose Taylor coefficients satisfy $(2.14)$, for any prescribed $\beta<1 / 2$, with infinitely many zeroes in $\mathbb{D}$.

Proof of Theorem 2.1. Note that (2.6) and (2.7) imply the absolute convergence of each of the series (2.8). Consider now the function

$$
g(x)=\sum_{n=1}^{\infty} c_{n} \cos \left(\lambda_{n}^{1 / 2} x\right), \quad x \in \mathbb{R}
$$

In view of (2.7), $g$ extends as an analytic function of $z=x+i y$ into a strip $\{z:|\operatorname{Im} z|<\delta\}$ for some $\delta>0$. Then (2.8) expresses the fact that all even-order derivatives of $g$ vanish at $z=0$. Since $g$ is an even function, $g \equiv 0$. Now, $g(x)$ is the Fourier-Stieltjes transform of the discrete measure which places masses $c_{n} / 2$ at points $\pm \lambda_{n}^{1 / 2}$. By 
the uniqueness theorem for Fourier-Stieltjes transforms this measure vanishes, i.e. all $c_{n}$ are zero. This concludes the proof.

REMARK. The hypothesis $\left|c_{n}\right| \leq e^{-\varepsilon \lambda_{n}^{1 / 2}}$ in Theorem 2.1 could be weakened. What is essential is that $c_{n}$ are small enough so that

$$
\sum c_{n} \cos \left(\lambda_{n}^{1 / 2} x\right)
$$

falls into a quasi-analytic class on $\mathbb{R}$, in the sense of Denjoy-Carleman. One knows precisely what decay of $\left\{c_{n}\right\}$ is necessary for this, $c f$. [M]. We shall not however pursue this kind of generalization, which involves only well-known ideas.

Carleson [Ca2] obtains Corollary 1 in a somewhat different manner. He introduces

$$
\varphi(s)=\sum_{n=1}^{\infty} c_{n} n^{s}
$$

which is clearly an entire function of $s$ under the hypothesis (2.9). It is easy to see (2.9) implies the estimate

$$
\log |\varphi(\sigma+i \tau)| \leq \frac{2}{p} \sigma \log \sigma+O(\sigma)
$$

for $\sigma>0$. He now applies the following theorem, for which see [Ca1]:

If $\varphi$ is analytic in the right half-plane and satisfies

$$
|\varphi(\sigma+i \tau)| \leq C e^{m(\sigma)}
$$

where $m(\sigma)$ is convex on $\mathbb{R}^{+}$and for some $p>0$

$$
\int_{1}^{\infty} \exp ((-p / 2) m(\sigma) / \sigma) d \sigma=\infty
$$

and

$$
\varphi(p k)=0, \quad k=0,1,2, \ldots
$$

then $\varphi \equiv 0$. 
To obtain Corollary 1 from this one uses (2.18) to verify that (2.19) and (2.20) hold, and (2.21) is just (2.10); hence $\varphi \equiv 0$, which easily implies that all $c_{n}$ vanish.

The theorem employed by Carleson is known to be sharp, but that does not imply the sharpness of Corollary 1 because a function satisfying an estimate (2.19) is not necessarily representable as a Dirichlet series (2.17).

Since the theorem is only stated, but not proved in [Ca1], we refer the reader to [Mal, pp. 184-185] for a proof.

\section{An example of non-uniqueness and some of its ramifica- tions.}

Theorem 3.1. For any $p>2$, writing $\lambda_{n}=n^{p}(n \geq 0)$, there exists a complex sequence $\left\{c_{n}\right\}$ satisfying

$$
\varlimsup_{n \rightarrow \infty}\left|c_{n}\right|^{1 / n}=\delta_{p}=\exp \left(-\pi \operatorname{ctg} \frac{\pi}{p}\right)
$$

such that

$$
f(z)=\sum_{n=0}^{\infty} c_{n} e^{-\lambda_{n} z}
$$

(which converges for $\operatorname{Re} z \geq 0$, and extends as a $C^{\infty}$ function to the closed right half-plane) has an infinite-order zero at $z=0$. In other terms,

$$
\sum_{n=0}^{\infty} c_{n} n^{p k}=0, \quad k=0,1,2, \ldots
$$

Moreover, for positive $x$

$$
|f(x)| \leq C \exp \left(-c x^{-1 / p}\right)
$$

where $C, c$ are positive constants.

For integral $p$, the constant on the right side of (3.1) is sharp, in the sense that no such sequence $\left\{c_{n}\right\}$ exists with $0<\varlimsup_{n \rightarrow \infty}\left|c_{n}\right|^{1 / n}<\delta_{p}$. 
We postpone the proof, and discuss some consequences of the theorem. Let $p \geq 3$ be an integer, and let $\left\{c_{n}\right\}$ be as in the theorem. As in Section 1, form the $p$-fold symmetrization of the function

$$
g(z)=\sum_{n=0}^{\infty} c_{n} e^{-n z}
$$

about the origin. We may denote this by $F(z ; p)$.

Since the $p$-fold symmetrization about 0 of $e^{z}$ is $\sum_{k=0}^{\infty} z^{p k} /(p k) !$, one computes easily

$$
\begin{aligned}
F(z ; p) & =\sum_{n=0}^{\infty} c_{n} \sum_{k=0}^{\infty} \frac{(-n z)^{p k}}{(p k) !} \\
& =\sum_{k=0}^{\infty} \frac{z^{p k}}{(p k) !} \sum_{n=0}^{\infty} c_{n}(-n)^{p k}=0
\end{aligned}
$$

in view of (3.3). We thus have

Corollary. For any integer $p \geq 3$ there exists a Dirichlet series (3.5) whose coefficients satisfy (3.1) (and hence: $g$ is analytic in a half-plane $\{\operatorname{Re} z \geq-\delta\}$ for some $\delta>0$ ) whose $p$-fold symmetrization about the origin vanishes identically. In other terms,

$$
\sum_{k=0}^{p-1} \sum_{n=0}^{\infty} c_{n} \exp \left(-\omega^{k} n z\right) \equiv 0, \quad \omega=e^{2 \pi i / p} .
$$

Remark. Note that (3.6) is a Dirichlet series of general type whose "exponents" are the set $\left\{-\omega^{k} n: 0 \leq k \leq p-1, n \in \mathbb{N}\right\}$ which is distributed along $p$ rays through the origin. Condition (3.1) guarantees that this series converges absolutely on a neighborhood of $z=0$, yet not in the whole plane. But the sum is an entire function (indeed, zero!). This behaviour is in stark contrast with the cases $p=1$ (Taylor series in $e^{-z}$ ) and $p=2$ (Laurent series in $e^{-z}$ ). Recalling our discussion of Fredholm's method in Section 1, we see that (1.9) could in fact be entire, subject to $(1.2) \ldots$ so this method encounters an unforeseen difficulty when applied to a series with gaps like $\sum a_{n} \zeta^{n^{3}}$. (Thus, Mittag-Leffler's opinion that Fredholm's method could be generalized may be too optimistic; however, some gap series of type $\sum a_{n} \zeta^{n^{3}}$ can be exhibited by Fredholm's method by requiring $\varlimsup\left|a_{n}\right|^{1 / n}$ suitably small, see the discussion following Theorem 4.1 below.) 
Proof of Theorem 3.1. The proof is based on a construction that has been used previously by Hirschman and Jenkins [HJ1], [HJ2], Anderson $[\mathrm{A}]$ and others for somewhat different purposes. Let

$$
\varphi(w)=\prod_{n=1}^{\infty}\left(1+\frac{w}{n^{p}}\right) .
$$

Clearly $\varphi$ is an entire function. By estimates given later, we will show it has order $1 / p$, and moreover that

$$
f(x)=(2 \pi i)^{-1} \int_{\gamma} \varphi(w)^{-1} e^{x w} d w
$$

where $x \in \mathbb{R}$, and $\gamma$ denotes the imaginary axis traversed from $-\infty$ to $+\infty$, is an absolutely convergent integral; and that translating $\gamma$ parallel to itself (to a position that does not contain a point $-n^{-p}$ $(n \in \mathbb{N}))$ preserves convergence, and changes the integral only by the sum of residues of the poles passed over. Moving the contour leftwards to the position

$$
\gamma_{m}=\left\{\operatorname{Re} w=-\left(\lambda_{m} \lambda_{m+1}\right)^{1 / 2}\right\}
$$

where for convenience we denote

$$
\lambda_{m}=m^{-p}
$$

and letting $m \rightarrow \infty$ gives, formally,

$$
f(x)=\sum_{n=1}^{\infty} \varphi^{\prime}\left(-\lambda_{n}\right)^{-1} e^{-\lambda_{n} x} .
$$

As we will show later, for $n>n_{0}$ we have

$$
\log \left|\varphi^{\prime}\left(-\lambda_{n}\right)\right| \sim(\pi \operatorname{ctg}(\pi / p)+o(1)) n
$$

as $n \rightarrow \infty$, and so

$$
f(z)=\sum_{n=1}^{\infty} \varphi^{\prime}\left(\lambda_{n}\right)^{-1} e^{-\lambda_{n} z}
$$


converges uniformly for $z$ on compact subsets of $\{\operatorname{Re} z>0\}$. We shall show that this function $f$ satisfies the requirements of the theorem. Thus, $c_{n}=\varphi^{\prime}\left(\lambda_{n}\right)^{-1}$, and (3.12) implies (3.1).

We will first verify (3.4) which, since clearly $f$ is $C^{\infty}$ on the closed right half-plane, implies (3.3) (of course (3.4) is much stronger than (3.3)). Fix $x>0$ in (3.8) and move $\gamma$ to the right, to $\left\{w: \operatorname{Re} w=x^{-1}\right\}$. A crude estimate gives

$$
|f(x)| \leq \frac{e}{2 \pi} \int_{(1 / x)-i \infty}^{(1 / x)+i \infty}|\varphi(w)|^{-1}|d w|
$$

and to get (3.4) from this we require a lower bound for $|\varphi(w)|$. We have for $\operatorname{Re} w=u>0$,

$$
|\varphi(w)|=|1+w|\left|1+2^{-p} w\right| \prod_{n=3}^{\infty}\left|1+n^{-p} w\right|
$$

and the infinite product is not less than

$$
\prod_{n=3}^{\infty}\left(1+n^{-p} u\right) \geq \prod_{3 \leq n \leq u^{1 / p}}\left(n^{-p} u\right) \geq(N !)^{-p} u^{N-3}
$$

where $N$ denotes the least integer $\geq u^{1 / p}$. Simple estimates based on Stirling's formula show the last expression exceeds $\exp \left(p u^{1 / p}-c \log u\right)$ for some positive $c$ (henceforth $c, c_{1}, \ldots$ will designate positive constants whose precise value is of no concern). Hence, from (3.15),

$$
|\varphi(u+i v)| \geq c_{1}\left(1+v^{2}\right) \exp \left((p / 2) u^{1 / p}\right)
$$

and inserting this in (3.14) (with $u=1 / x$ ) gives (3.4).

We turn now to the estimate (3.12). From (3.7),

$$
\varphi^{\prime}\left(-\lambda_{n}\right)=n^{p} \prod_{\substack{m=1 \\ m \neq n}}^{\infty}\left(1-\frac{n^{p}}{m^{p}}\right)
$$

Now,

$$
\log \prod_{\substack{m=1 \\ m \neq n}}^{\infty}\left|1-\frac{n^{p}}{m^{p}}\right|=\sum_{\substack{m=1 \\ m \neq n}}^{\infty} \log \left|1-\frac{n^{p}}{m^{p}}\right|=\sum_{\substack{m=1 \\ m \neq n}}^{\infty} \psi\left(\frac{m}{n}\right)
$$


where

$$
\psi(t)=\log \left|1-t^{-p}\right|, \quad t>0 .
$$

Note that for $p>1$ the improper Riemann integral of $\psi$ over $(0,+\infty)$ exists, and since $\psi$ is piecewise monotone (decreasing on $(0,1)$, increasing on $(1,+\infty))$ it is easy to verify that the Riemann sums

$$
n^{-1} \sum_{\substack{m=1 \\ n \neq n}}^{\infty} \psi\left(\frac{m}{n}\right)
$$

converge to $A_{p}=\int_{0}^{\infty} \psi(t) d t$. Thus

$$
\sum_{\substack{m=1 \\ m \neq n}}^{\infty} \psi\left(\frac{m}{n}\right) \sim A_{p} n, \quad \text { as } n \rightarrow \infty,
$$

So

$$
\prod_{\substack{m=1 \\ m \neq n}}^{\infty}\left|1-\frac{n^{p}}{m^{p}}\right|=\exp \left(\left(A_{p}+o(1)\right) n\right)
$$

which yields (3.12) if we verify that the improper Riemann integral

$$
A_{p}=\int_{0}^{\infty} \log \left|1-t^{-p}\right| d t
$$

has the value $\pi \operatorname{ctg}(\pi / p)$. For this, see [GH, p. 84, formula 8a]. This proves (3.12).

To conclude the proof of the theorem, we now derive the estimates for $\varphi$ that were needed to justify moving the contour of integration in (3.8). These are well known ( $c f$. [Boa, p. 19]), but for the reader's convenience we present the details since some of the intermediate estimates will be required. We first study $\varphi$ in $\mathbb{C} \backslash \bar{\Omega}_{\beta}$ where $\beta<\pi / 2$ and

$$
\Omega_{\beta}=\{z:|\pi-\arg z|<\beta\} .
$$

In $\mathbb{C} \backslash \bar{\Omega}_{\beta}, \log \varphi$ has a single-valued analytic branch that is real on the positive real axis. In the following calculation, we work with this branch, and restrict $z$ to $\mathbb{C} \backslash \bar{\Omega}_{\beta}$.

$$
\log \varphi(z)=\sum_{n=1}^{\infty} \log \left(1+\frac{z}{n^{p}}\right)=\int_{0+}^{\infty} \log \left(1+t^{-p} z\right) d[t]
$$


where [.] denotes the greatest integer function. Applying partial integration to the last integral gives

$$
\begin{aligned}
\log \varphi(z) & =p z \int_{1}^{\infty} \frac{[t]}{t} \frac{d t}{z+t^{p}} \\
& =p z \int_{1}^{\infty} \frac{d t}{z+t^{p}}+O\left(|z| \int_{1}^{\infty} \frac{d t}{t\left|z+t^{p}\right|}\right) .
\end{aligned}
$$

The first integral on the right can be evaluated by applying Cauchy's theorem. First, observe that

$$
\int_{1}^{\infty} \frac{d t}{z+t^{p}}=\int_{0}^{\infty} \frac{d t}{z+t^{p}}+O\left(|z|^{-1}\right), \quad|z| \rightarrow \infty
$$

and, writing $z=r e^{i \theta}$, we move the line of integration in the right-hand integral to $\{\arg t=\theta / p\}$, so that $t=s e^{i \theta / p}, s>0$. We get

$$
\int_{0}^{\infty} \frac{d t}{z+t^{p}}=\left(\exp i\left(\frac{1}{p}-1\right) \theta\right) \int_{0}^{\infty} \frac{d s}{r+s^{p}}
$$

which after some simplification becomes $C_{p}\left(r e^{i \theta}\right)^{-1+1 / p}$, where $C_{p}$ is a positive constant $\left(C_{p}=\int_{0}^{\infty}\left(1+u^{p}\right)^{-1} d u\right)$. Thus, the first term on the right of (3.21) is $C^{\prime} z^{1 / p}+O(1)$ for large $z \in \mathbb{C} \backslash \bar{\Omega}_{\beta}$. We will now show that the second term in (3.21) is of smaller order. This will establish:

$$
\varphi(z) \sim \exp \left(C_{p}^{\prime} z^{1 / p}\right)
$$

holds for large $z$ outside each sector symmetric with respect to the negative real axis (where $C_{p}^{\prime}$ is a positive constant depending only on $p$ ). In particular, $\varphi$ is of order $1 / p$. Observe that (3.22) gives the rapid decrease of $|\varphi(x+i y)|^{-1}$ as $|y| \rightarrow \infty$ which was required for moving the line of integration since, from (3.22) (with $x+i y=z=r e^{i \theta}$ ), we get

$$
|\varphi(z)| \sim \exp \left(C_{p}^{\prime} \cos (\theta / p) r^{1 / p}\right), \quad z \in \mathbb{C} \backslash \bar{\Omega}_{\beta}
$$

and, since $p>2, \cos (\theta / p)$ is positive for $|\theta| \leq \pi$.

Now we estimate the $O$-term in (3.21). Consider separately the cases $x \geq 0$ and $x<0$.

For $x \geq 0,\left|z+t^{p}\right|^{2} \geq|z|^{2}+t^{2 p}$, so

$$
|z| \int_{1}^{\infty} \frac{d t}{t\left|z+t^{p}\right|} \leq|z| \int_{1}^{\infty} \frac{d t}{t\left(t^{2 p}+|z|^{2}\right)^{1 / 2}} \leq C \log (1+|z|)
$$


while for $\operatorname{Re} z<0, z \in \mathbb{C} \backslash \bar{\Omega}_{\beta}$ we have

$$
\left|z+t^{p}\right| \geq c|z|, \quad c=c(\beta) .
$$

Hence

$$
\int_{1}^{\infty} \frac{d t}{t\left|z+t^{p}\right|}=\left(\int_{1}^{T}+\int_{T}^{\infty}\right) \frac{d t}{t \mid z+t^{p \mid}}
$$

(where $\left.T=(2|z|)^{1 / p}\right)$, which is

$$
\leq \int_{1}^{T} \frac{d t}{c|z| t}+\int_{T}^{\infty} \frac{d t}{t(1 / 2) t^{p}}
$$

(since $\left|z+t^{p}\right| \geq t^{p}-|z| \geq(1 / 2) t^{p}$ for $\left.t \geq T\right)$,

$$
=(c|z|)^{-1} \log T+O\left(T^{-p}\right)=O\left(|z|^{-1} \log |z|\right)
$$

for large $|z|$. Hence, the $O$-term in (3.21) is $O(\log |z|)$ for large $|z|$ outside $\Omega_{\beta}$, and (3.22) is completely proved.

To conclude the proof of our theorem we need only verify one last point: that the integral (3.8) tends to zero as $\gamma$ is moved sufficiently far to the left, since that was assumed in the passage from (3.8) to (3.11). For this purpose we recall that, since $\varphi$ is of order $1 / p<1 / 2$ there is a sequence $R_{j} \rightarrow \infty$ such that

$$
\log m\left(R_{j}\right)>\cos (\pi / p) \log M\left(R_{j}\right)
$$

where $m(R), M(R)$ denote the minimum and maximum of $|\varphi(w)|$ on $\{|w|=R\}$, respectively (see [Boa, p. 40, Theorem. 3.1.6]). Thus, we may move $\gamma$ leftwards in (3.8) through the sequence $\gamma_{j}$, where (for some fixed $\beta$, say $\beta=\pi / 4) \gamma_{j}$ consists of an arc of $\left\{|w|=R_{j}\right\}$ inside $\Omega_{\beta}$, completed by vertical half-lines outside $\Omega_{\beta}$. It follows at once from (3.23) and (3.24) that $\int_{\gamma_{j}}|\varphi(w)|^{-1}|d w| \rightarrow 0$ as $j \rightarrow \infty$. This completes the proof of Theorem 3.1, apart from the sharpness of the constant in (3.1) to which point we shall return in the next Section. 


\section{A result implying existence of singularities.}

We saw, in the Corollary to Theorem 3.1, that if $p$ is an integer, $p \geq 3$, there exist complex $\left\{c_{n}\right\}$ with

$$
0<\varlimsup_{n \rightarrow \infty}\left|c_{n}\right|^{1 / n}=\delta<1
$$

such that

$$
g(z)=\sum_{n=0}^{\infty} c_{n} e^{-n z}
$$

(the series converges absolutely to a function holomorphic on a neighborhood of 0 ) whose $p$-fold symmetrization about 0 ,

$$
F(z ; p)=\frac{1}{p} \sum_{k=0}^{p-1} g\left(\omega^{k} z\right), \quad \omega=e^{2 \pi i / p},
$$

vanishes identically (and hence, is analytically continuable to all of $\mathbb{C}$ ). We now show that for small enough $\delta$ in (4.1), this cannot happen:

Theorem 4.1. Let $p \geq 3$ be an integer and suppose $\left\{c_{n}\right\}$ satisfy (4.1) with

$$
\delta<\delta_{p}=\exp (-\pi \operatorname{ctg}(\pi / p)) .
$$

Then $F(z ; p)$ does not extend to all of $\mathbb{C}$ without singularities; in fact, it has a singularity in the disk centered at 0 of radius $\left(\pi^{2}+(\log (1 / \delta))^{2}\right)^{1 / 2}$.

Observe that this implies the assertion in Theorem 3.1 concerning the sharpness of the constant. We do not know whether it is sharp also for non-integral $p$.

Before giving the proof, we observe a consequence of the theorem: for $\left\{c_{n}\right\}$ satisfying (4.1) with $\delta<\delta_{p}$ the power series $\sum_{0}^{\infty} c_{n} \zeta^{n^{p}}$ is not continuable across any point of $\partial \mathbb{D}$; this follows by the (modified) Fredholm argument we presented in Section 1. Of course, this argument has the blemish that the upper bound imposed on $\delta$ is purely fortuitous; one could remove it by combining the argument given with Hadamard's multiplication of singularities theorem and a few other things (see [KS] for details). 
Proof of Theorem 4.1. Observe that (4.2) converges absolutely for $\operatorname{Re} z>-\sigma$, where

$$
\sigma=\log \frac{1}{\delta}
$$

and since $g$ has period $2 \pi i$, it must have a singularity at a point $z_{0}=$ $-\sigma+i y_{0}$ for some $y_{0}$ with $-\pi<y_{0} \leq \pi$. Let $L$ denote the line segment joining 0 to $z_{0}$. It is clear that if $\left\{\omega^{k} z_{0}: k=1,2, \ldots, p-1\right\}$ all lie in $\{\operatorname{Re} z>-\sigma\}$, the analytic continuation of $F(z ; p)$ from 0 to $z_{0}$ along $L$ is possible as far as $z_{0}$, and encounters a singularity at $z_{0}$, since each $g\left(\omega^{k} z\right)$ for $1 \leq k \leq p-1$ is analytic on a neighborhood of the closure of $L$. And it is geometrically obvious that this occurs if the angle subtended by the points $-\sigma \pm i y_{0}$ at 0 is less than $2 \pi / p$. Since this angle can not exceed $2 \operatorname{arctg}(\pi / \sigma)$, we will have a singularity of $F(z ; p)$ at $z_{0}$ if

$$
2 \operatorname{arctg} \frac{\pi}{\sigma}<\frac{2 \pi}{p}
$$

i.e. if $\sigma>\pi \operatorname{ctg}(\pi / p)$, and in view of (4.4) this completes the proof.

\section{The integral analogue.}

Corollary 1 to Theorem 2.1 has an integral analogue: If

Let $f$ be a complex-valued continuous function $[0,+\infty)$ and $p>0$.

$$
|f(x)| \leq C \exp \left(-c x^{p / 2}\right)
$$

for some positive constants $C, c$ and

$$
\int_{0}^{\infty} f(x) x^{p k} d x=0, \quad k=0,1,2, \ldots
$$

then $f \equiv 0$.

The proof is similar to that given in the discrete case, and may be left to the reader. As before, we are mainly interested in examples to show the sharpness of the condition (5.1), and shall prove: 
Theorem 5.1. Given any $p>0$ and $0<q<p / 2$ there is a continuous $f$ on $[0, \infty], f \not \equiv 0$, satisfying (5.2) and

$$
|f(x)| \leq C \exp \left(-x^{q}\right), \quad x>0 .
$$

Proof. As is well known, for $0<b<1$ there is a non-null entire function $F$ of exponential type satisfying

$$
|F(x)| \leq e^{-|x|^{b}}, \quad x \in \mathbb{R} .
$$

The Fourier transform $\widehat{F}$ of $F$ (which is infinitely differentiable) has compact support. Multiplying $F$ by a suitable exponential $e^{i \lambda z}$ we can arrange that $\widehat{F}$ vanishes on a neighborhood of 0 , and that the even part of $F$,

$$
F_{e}(x)=\frac{F(x)+F(-x)}{2}
$$

does not vanish identically; we assume this is done. Since all derivatives of $\widehat{F}$ vanish at 0 ,

$$
\int_{-\infty}^{\infty} F(x) x^{n} d x=0, \quad n=0,1, \ldots
$$

hence

$$
\int_{0}^{\infty} F_{e}(x) x^{2 k} d x=0, \quad k=0,1,2, \ldots
$$

Changing variables in (5.6),

$$
\int_{0}^{\infty} F_{e}\left(t^{p / 2}\right) t^{p k} t^{p / 2-1} d t=0, \quad k=0,1, \ldots
$$

Letting $f(t)=t^{p / 2-1} F_{e}\left(t^{p / 2}\right)$ and observing (5.4), it is clear that $f$ satisfies (5.3) if $b$ is chosen greater than $2 q / p$. This completes the proof.

REMARKs. The idea to look at the integral analogue was suggested to us by D. J. Newman, who also provided an elegant proof of a weaker variant of Theorem 6.1, which we here sketch briefly. Starting from

$$
\Gamma(n p)=\int_{0}^{\infty} e^{-t} t^{n p-1} d t
$$


where $p>2$, rotate the line of integration to $\{\arg t=\pi / p\}$ giving

$$
\Gamma(n p)=(-1)^{n} \int_{0}^{\infty} \exp \left(-e^{i \pi u / p}\right) u^{n p-1} d u
$$

whence, taking imaginary parts

$$
\int_{0}^{\infty} e^{-\cos \pi u / p} \frac{\sin (\sin (\pi u / p))}{u} u^{n p} d u=0
$$

holds for $n=0,1,2, \ldots$ Thus, writing $a=\cos (\pi / p)>0, b=\left(1-a^{2}\right)^{1 / 2}$ we see that, setting

$$
\begin{gathered}
f(u)=e^{-a u}\left(\frac{\sin (\sin b u)}{u}\right) \\
\int_{0}^{\infty} f(u) u^{n p} d u=0, \quad \text { for } n=0,1, \ldots
\end{gathered}
$$

This gives $f$ which is precisely the continuous analogue of the sequence $\left\{c_{n}\right\}$ we constructed in Theorem 3.1: it decays exponentially on $\mathbb{R}^{+}$ and the moments (5.9) vanish. But for fixed $p>2$, this result is weaker than Theorem 5.1 (compare (5.8) with (5.3)). Moreover, the method we used to prove Theorem 5.1 can be made to yield more, since $F$ could be chosen to satisfy not merely (5.4), but

$$
|F(x)| \leq \exp (-\varphi(|x|))
$$

where $\varphi$ is any sufficiently regular positive increasing function on $\mathbb{R}^{+}$ with

$$
\int_{0}^{\infty} \frac{\varphi(t)}{1+t^{2}} d t<\infty
$$

Since these ideas are very well known, we do not pursue the details.

It would be interesting to extend Theorem 3.1 to the discrete analogue of Theorem 5.1, but we do not know how to do this.

Acknowledgements. We wish to thank James Clunie for stimulating discussions of this material. He also discovered Theorem 4.1 independently. We also thank Donald Newman, who suggested the topic of Section 5, and Lennart Carleson and Håkan Hedenmalm for references to the literature. 
The second author acknowledges with thanks financial assistance from the Göran Gustafsson foundation. The work was carried out while the first and second authors were visitors at the Royal Institute of Technology, and they express their gratitude to that institution for support.

\section{References.}

[A] Anderson, J. M. Bounded analytic functions with Hadamard gaps. Mathematika 23 (1976), 142-146.

[Be] Beurling, A., Sur les fonctions limites quasi analytiques des fractions rationelles. Proc. 8th Scandinavian Math. Congr., Stockholm (1934), 199-210.

[Boa] Boas, R. P., Entire Functions. Academic Press, 1954.

[BS] Bony, J. M. and Shapira, P., Existence et prolongement des solutions holomorphes des équations aux dérivées partielles. Invent. Math. 17 (1972), 95-105.

[Bor] Borel, E., Leçons sur la Théorie de Fonctions. Gauthier-Villars, 1898.

[BSZ] Brown, L., Shields, A. and Zeller, K., On absolutely convergent exponential sums. Trans. Amer. Math. Soc. 96 (1960), 162-183.

[C] Carleman, T., Sur les séries $\sum A_{r}\left(z-\alpha_{r}\right)^{-1}$. C. R. Acad. Sci. Paris 174 (1922), 588-591.

[Ca1] Carleson, L., On Bernstein's approximation problem. Proc. Amer. Math. Soc. 2 (1951), 953-961.

[Ca2] Carleson, L., Sets of uniqueness for functions regular in the unit circle. Acta Math. 87 (1952), 325-345.

[D] Denjoy, A., Sur les singularités des séries de fractions rationelles. Rendiconti del Circolo Mat. di Palermo 50 (1926), 1-95.

[Di] Dienes, P., The Taylor Series. Oxford Univ. Press, 1931.

[F] Fredholm, I., Om en speciell klass af singuljara linjer. Öfv. Kungl. Svenska Vetensk. Akad. Förh. 3 (1890), 32-34. Reproduced in Oeuvres completes de Ivar Fredholm, Institut Mittag-Leffler (1955), 1-5.

[GH] Gröbner, W. and Hofreiter, N., Integraltafel, Zweiter Teil (Bestimmte Integrale). Springer-Verlag, 1958.

[Ha] Hadamard, J., Lectures on Cauchy's Problem in Linear Partial Differential Equations. Reprinted by Dover Publications, 1952.

[HJ1] Hirschman Jr., I. and Jenkins, J., Note on a result of Levine and Lifschitz. Proc. Amer. Math. Soc. 1 (1950), 390-393.

[HJ2] Hirschman Jr., I. and Jenkins, J., On lacunary Dirichlet series. Proc. Amer. Math. Soc. 1 (1950), 512-517. 
[H] Hörmander, L., The analysis of Linear Partial Differential Operators I. Springer-Verlag, 1983.

[KS] Khavinson, D. and Shapiro, H. S., The heat equation and analytic continuation: Ivar Fredholm's first paper. Expositiones Math. 12 (1994), 79-95.

[Ki] Kiselman, C., Prolongement des solutions d'une équation aux dérivées partielles à coefficients constants. Bull. Soc. Math. France 97 (1969), 329-356.

[Ko] Kovalevskaya, S., Zur Theorie der partiellen Differentialgleichungen. J. Reine und Angew. Math. 80 (1875), 1-32.

[L] Leontiev, A. F., Series of Exponentials. Nauka, 1976 (Russian).

[Mal] Malliavin, P., Sur quelques procédés d' extrapolation. Acta Math. 93 (1955), 179-255.

[M] Mandelbrojt, S., Séries de Fourier et classes quasianalytiques de fonctions. Paris, 1935.

[ML] Mittag-Leffler, G., Sur une transcendante remarquable trouvée par M. Fredholm. Acta Math. 15 (1891), 279-280.

[TW] Taylor, B. A. and Williams, D., Ideals in rings of analytic functions with smooth boundary values. Canad. J. Math. 22 (1970), 1266-1283.

[Ti] Titchmarsh, E. C., The Theory of Functions. Oxford Univ. Press, 1939.

[W] Wolff, J., Sur les séries $\sum A_{k} /\left(z-\alpha_{k}\right)$. C. R. Acad. Sci. Paris 173 (1921), 1057-1058, and 1327-1328.

Recibido: 7 de febrero de 1.994

Revisado: 14 de septiembre de 1.994

J. Milne Anderson

Department of Mathematics

University College London

Gower Street

London WC1E 6BT

UNITED KINGDOM

ros@math.ucl.ac.uk
Dimitry Khavinson

Department of Mathematics

University of Arkansas

Fayetteville, AR 72701-1201

U.S.A.

dk24653@uaf sysb . uark . edu

and

Harold S. Shapiro

Department of Mathematics

Royal Institute of Technology

S-100 44 Stockholm, SWEDEN

shapiro@math.kth.se 